TRABAJOS ORIGINALES

\title{
Estudio genotóxico de una bebida experimental de quinua, kiwicha y kañiwa
}

\author{
Genotoxicity study of an experimental beverage made with quinua, kiwicha and kañiwa
}

\author{
Francia D.P. Huaman ${ }^{1}$, Emily M. Toscano ${ }^{2}$, Oscar Acosta ${ }^{1}$, Diana E. Rojas ${ }^{1}$, Miguel A. Inocente ${ }^{2}$, Diana P. \\ Garrido $^{2}$, María L. Guevara-Fujita ${ }^{1 *}$
}

(1) Centro de Genética y Biología Molecular (CGBM), y (2) Centro de Medicina Tradicional (CMT). Instituto de Investigación de la Facultad de Medicina Humana de la Universidad San Martín de Porres. Av. Alameda del Corregidor N¹531. Urb. Los Sirius III Etapa. La Molina, Lima 12, Perú. * Autor para correspondencia

María L. Guevara-Fujita. Los Tucanes 296, San Isidro, Lima 27, Perú.

Email Francia Huaman: franciahd19@gmail.com Email Emily Toscano: ema.tg27@gmail.com Email Oscar Acosta: oacostac@yahoo.com Email María Guevara: mguevarag@usmp.pe Email Diana Rojas: diana.rojas.malaga@gmail.com Email Miguel Inocente: minocente@farmaceuticos.com Email Diana Garrido: dgarridom@usmp.pe

Citación:

Huaman F.D.P. , E.M. Toscano, O. Acosta, D.E. Rojas, M.A. Inocente, D.P. Garrido, M.L. Guevara-Fujita. 2014. Estudio genotóxico de una bebida experimental de quinua, kiwicha y kañiwa. Revista peruana de biología 21(3): 251 - 258 (Diciembre 2014). doi: http://dx.doi.org/10.15381/ rpb.v21i3.10899

Fuentes de financlamlento:

El presente trabajo fue financiado por:

Proyecto CONCYTEC - OAJ 368-2012. Facultad de Medicina Humana- Universidad de San Martín de Porres. Proyecto E10012014001.

\begin{abstract}
Resumen
La evaluación genotóxica de un producto es un paso importante para determinar su viabilidad para consumo humano. Se ha elaborado una bebida experimental a base de pseudocereales de alto valor nutricional como son quinua (Chenopodium quinoa Willd.), kiwicha (Amaranthus caudatus L.) y kañiwa (Chenopodium pallidicaule Aellen), preparada para inducir un posible efecto hipolipemiante en un grupo de personas. El objetivo de este estudio fue evaluar el potencial genotóxico de esta bebida experimental mediante dos pruebas in vitro validadas por agencias internacionales. En la prueba de Ames se utilizaron las cepas TA98 y TA100 de Salmonella typhimurium, con y sin fracción microsomal (S9). Se evaluaron 4 dosis de bebida y además un posible efecto antimutagénico (mismas 4 dosis más mutágeno). Para la prueba de micronúcleos se usó cultivos de linfocitos con células binucleadas, en presencia de cinco dosis de la bebida. Ambas pruebas indican que la bebida estudiada en sus distintas dosis, no presenta efecto genotóxico. Sin embargo, en la evaluación del posible efecto protector de la bebida, se evidenciaría que por el contrario, se potencia el efecto mutagénico de los mutágenos empleados para cada cepa. Por lo tanto, es importante que esta bebida experimental sea sometida a pruebas adicionales in vitro e in vivo para evaluar el potencial genotóxico antes de su consumo.
\end{abstract}

Palabras clave: Quinua; Kiwicha; Kañiwa; Prueba de Ames; Prueba de Micronúcleos.

\section{Abstract}

Genotoxic evaluation is an important step for a product that is aimed for human consumption. A beverage composed of pseudocereals with highly nutritious elements like quinua (Chenopodium quinoa Willd.), kiwicha (Amaranthus caudatus L.) and kañiwa (Chenopodium pallidicaule Aellen) was prepared to reduce lipid contents in a group of volunteers. The objective of the present study is to evaluate the genotoxic potential of an experimental beverage using two in vitro tests that have been validated by international agencies. For the Ames test, two strains of Salmonella typhimurium (TA98 and TA100) with and without microsomal fraction (S9) were used. Four doses of the beverage were tested and also a possible protective effect (same four doses of beverage added to plates with mutagens). Cultures of binucleated lymphocytes and five doses of the beverage were used for the micronucleus test. Both Ames and the micronucleus tests showed the beverage has not genotoxic effect in all tested doses. However, in evaluating the possible protective effect of the beverage, it would be evident that on the contrary, the mutagenic effect of mutagens used for each strain is enhanced. These results suggest that additional tests should be performed to check the genotoxic potential of this beverage before consumption.

Keywords: Quinua; Kiwicha; Kañiwa; Ames test; Micronucleus test

Informacion sobre los autores:

Los autores declaran no tener conflicto de intereses sobre la investigación y el artículo a publicar.

$\begin{array}{ll}\text { Presentado: } & 02 / 06 / 2014 \\ \text { Aceptado: } & 25 / 11 / 2014 \\ \text { Publicado online: } & 30 / 12 / 2014\end{array}$

Journal home page: http://revistasinvestigacion.unmsm.edu.pe/index.php/rpb/index

(c) Los autores. Este artículo es publicado por la Revista Peruana de Biología de la Facultad de Ciencias Biológicas, Universidad Nacional Mayor de San Marcos. Este es un artículo de acceso abierto, distribuido bajo los términos de la Licencia Creative Commons Atribución-NoComercial-Compartirlgual 4.0 Internacional.(http://creativecommons.org/licenses/by-nc-sa/4.0/), que permite el uso no comercial, distribución y reproducción en cualquier medio, siempre que la obra original sea debidamente citadas. Para uso comercial, por favor póngase en contacto con editor.revperubiol@gmail.com. 


\section{Introducción}

La quinua (Chenopodium quinoa Willd.), kiwicha (Amaranthus caudatus L.)y kañiwa (Chenopodium pallidicaule Aellen) son granos andinos de consumo ancestral tradicional. La quinua se ha cultivado en una gran parte de la región andina de Perú, Bolivia y otros países (Carrasco et al. 2010); es conocida como el "cereal madre" y fue el alimento básico de las poblaciones precolombinas junto con la papa. Aunque se conoce el alto valor nutricional de este y otros cultivos andinos, no se ha logrado establecer su consumo masivo, principalmente debido a la escasa difusión de sus propiedades, las que permitirían suplir algunas de las necesidades nutricionales de la población. Además, se busca usar alimentos que no solo mejoren las condiciones físicas y mentales, sino que disminuyan el riesgo de contraer enfermedades y mejoren la calidad de vida (Turley et al. 2003). Estos granos andinos por su alto contenido proteico, según la FAO (Organización de las Naciones Unidas para la Alimentación y Agricultura) y la OMS (Organización Mundial de la Salud), son considerados como alimentos únicos por su altísimo valor nutricional. Además contienen compuestos polifenólicos como la quercitina, kaempfenol, genisteína y flavonoides como el epigalotequin galato (EGCG) (Tsuda et al. 2003).

Las enfermedades cardiometabólicas se vienen incrementando en la población y tienen un origen complejo y multifactorial, incluyendo niveles elevados de colesterol. Por ello, hay políticas de salud orientadas a la disminución de los niveles de colesterol, sobre todo en individuos afectados y/o con antecedentes familiares (Rossi et al. 2002; Huff 2003). El éxito de esta iniciativa es relativo, pues están involucrados factores como la biosíntesis, la reabsorción intestinal, la utilización metabólica, la edad, el sexo, y la genética (Altmann et al. 2004).

El Centro de Medicina Tradicional (CMT) de la Facultad de Medicina de la Universidad San Martín de Porres (FMHUSMP), ha elaborado una bebida experimental que contiene una mezcla de estos tres granos con la finalidad de evaluar un posible efecto hipolipemiante en voluntarios obesos (Proyecto 3682012-OAJ CONCYTEC) mediante la medición de los niveles de colesterol. Esta bebida contiene metabolitos (ej. flavonoides), se infiere con propiedades antioxidantes, antimicrobianas y por tanto con un impacto positivo en la prevención de la obesidad y disminución de la hiperglicemia, tal como se ha comprobado en ratones (Gálvez et al. 2009, Rastrelli et al. 1995).

Un tratamiento con esta bebida experimental puede llegar a ser muy eficaz en términos de ayudar a controlar los niveles de colesterol. Sin embargo, para validar su uso y aconsejar su consumo, es importante conocer si tiene efectos tóxicos y/o efectos negativos sobre el material genético en un sistema experimental.

Muchos estudios han relacionado el potencial genotóxico que un organismo o sistema celular puede tener, con la posibilidad de desarrollar cáncer. Esta correlación es importante cuando se utilizan plantas cuya composición química y posibles efectos se desconocen. En medicina tradicional se han descrito varias plantas con "riesgo mutagénico". Por ello, se hace necesario generar datos que provean información sobre la eficacia, calidad y seguridad de dichas plantas usadas tradicionalmente en nuestros países (Déciga-Campos et al., 2007; Verschaeve \& Van Staden 2008, Srivdya et al. 2013).
El potencial efecto genotóxico de numerosas sustancias de uso común en diferentes industrias es permanentemente evaluado para conocer su inocuidad. Desde hace varios años se han propuesto cientos de ensayos para evaluar genotoxicidad, y algunos, han sido aprobados por entidades internacionales, como la FDA (Agencia de Drogas y Alimentos), el CBER (Centro para la Evaluación e Investigación Biológica) entre otros. (European Medicines Agency 1996)

Las guías internacionales como las de EPA (Environmental Protection Agency) han aprobado sistemas in vitro de pruebas, como la de Ames en bacterias y micronúcleos en células binucleadas. Cada prueba evalúa el potencial genotóxico a diferentes niveles de posible daño al ADN (génico o cromosómico). (U.S Department of Health and Human Services Food and Drug Administration, Center for Drug Evaluation and Research (CDER) \& CBER 2012).

La prueba de Ames es una prueba microbiológica que utiliza diferentes cepas de Salmonella typhimurium (descrita por Loeffler 1892), auxotróficas a histidina, capaces de detectar mutaciones de cambio del marco de lectura o de sustitución de pares de bases. En esta prueba se pueden evaluar diferentes compuestos, ańadiéndolos al medio donde las bacterias crecen y se analiza como variable, el número de colonias revertantes, que se encuentra en proporción al efecto genotóxico de dichos compuestos (Maron \& Ames 1983). A esta prueba también se le puede incluir fracción microsomal de hígado de rata (S9), que imita el sistema metabólico en mamíferos, permitiendo la evaluación de posibles metabolitos secundarios derivados de procesos de transformación que normalmente no se dan en los sistemas bacterianos (Claxton et al. 1987). Hasta ahora, sólo se ha hecho el tamizaje del $7 \%$ de los químicos disponibles en el mercado. Según el New Chemical Program (U.S EPA, 2010), de los 50000 químicos pre-manufacturados, sólo $10 \%$ tienen datos de mutagenicidad (mayoritariamente Prueba de Ames). Esta prueba, por su simple desarrollo, flexibilidad y costo, constituye una de las más importantes para los ensayos genotoxicológicos (Mortelmans \& Zeiger 2000, Srividya et al. 2013).

El test de micronúcleos $(\mathrm{MN})$ detecta pérdidas cromosómicas debidas a defectos en la segregación de las cromátidas que llevan a la no disyunción o pérdida cromosómica (aneugénesis) o fragmentos originados por roturas de la cromatina (clastogénesis). Estos MN quedan excluídos de los núcleos de las células hijas en un cultivo que está en división activa. (Arencibia \& Rosario 2009, Zalacain et al. 2005). Según el grupo de trabajo denominado HUMN (Human Micronucleus Database in Human Lymphocytes) primero se debe evaluar el índice de división nuclear (IDN), cuyo valor óptimo está en el rango de 1.3-2.0 (Bonassi et al. 2001, Fenech et al. 2003), y posteriormente evaluar el índice de células binucleadas con/sin micronúcleos en cada muestra evaluada, estimándose el daño ocasionado en las células cultivadas. Esta prueba ha sido ampliamente validada e incorporada por las agencias internacionales (Bonassi et al 2001, Castillo et al. 2011).

No existe una sola prueba que determine exactamente el potencial mutagénico de una sustancia. Por ello, se puede usar más de una prueba para la evaluación. Es además reconocido, que cada prueba puede tener fortalezas pero también desventajas (Srividya et al. 2013). 
Tabla 1. Diseño experimental de la Prueba de Ames

\begin{tabular}{|c|c|c|c|c|c|}
\hline \multicolumn{3}{|c|}{ Prueba de mutagenicidad } & \multicolumn{3}{|c|}{ Prueba de antimutagenicidad } \\
\hline Experimento & Tratamiento* $(\mu \mathrm{L} /$ placa $)$ & Variable & Experimento & Tratamiento* $(\mu \mathrm{L} /$ placa $)$ & Variable \\
\hline \multicolumn{6}{|c|}{ Control Negativo $^{1}$} \\
\hline SIN S9 & Control Positivo² & \multirow{5}{*}{$\begin{array}{l}\text { \# De colonias } \\
\text { revertantes }\end{array}$} & \multirow{5}{*}{ SIN S9 } & Mutágeno(M) ${ }^{3}$ & \multirow{5}{*}{$\begin{array}{l}\text { \# De colonias } \\
\text { revertantes }\end{array}$} \\
\hline & Dosis 1: 10 & & & Dosis $1+\mathrm{M}^{3}$ & \\
\hline$y$ & Dosis 2: 100 & & & Dosis $2+\mathrm{M}^{3}$ & \\
\hline \multirow[t]{2}{*}{ CON S9 } & Dosis 3: 200 & & & Dosis $3+\mathrm{M}^{3}$ & \\
\hline & Dosis 4: 500 & & & Dosis $4+\mathrm{M}^{3}$ & \\
\hline
\end{tabular}

1 Cultivo al que no se le agregó nada.

2 Cultivo al que se le agregó, el 4-nitro-o-phenylenediamine (NPD) para la cepa TA98 y la azida de sodio para la cepa TA100 en experimentos sin S9, y el aminoantraceno para ambas cepas en experimentos con S9.

3 El mutágeno empleado es el mismo que en el caso del control positivo.

* Se realizaron tres pruebas con tres repeticiones

El presente estudio se realizó a fin de evaluar el posible efecto genotóxico de una bebida experimental producida a base de quinua, kiwicha y kañiwa por medio de dos pruebas in vitro de genotoxicidad.

\section{Materiales y métodos}

Muestra.- Se utilizó una bebida formulada y elaborada en el CMT FMH-USMP, en base a quinua (Chenopodium quinoa Willd.), kiwicha (Amaranthus caudatus L.)y kañiwa (Chenopodium pallidicaule Aellen). Se obtuvo la muestra después de 29 bebidas piloto, siendo todas estas sometidas a un análisis sensorial (sabor, aroma y color). La formulación seleccionada de acuerdo al panel de degustación fue el lote 103723, el cual fue sometido al control de calidad (análisis fisicoquímico). La composición elegida fue: $60 \%$ de agua, $20 \%$ de quinua, $10 \%$ kiwicha, 5\% de kańiwa y excipientes como la stevia, sorbato de potasio y vainillina (Inocente, et al. en preparación). La calidad microbiológica de la bebida se determinó mediante recuento de microorganismos aerobios, mohos y levaduras. Los parámetros fisicoquímicos, microbiológicos y de estabilidad, cumplieron con lo establecido en las normas e investigaciones consultadas. (Servicio de MICROBIOL S.A.).

Prueba de Ames.- Para esta prueba se utilizaron las cepas TA98 y la TA100 de S. typhimurium. Ambas fueron seleccionadas previo análisis de marcadores fenotípicos (mutación en el gen his de síntesis de histidina, inserción del plásmido pKM101(ApR) de resistencia a ampicilina, mutación en el gen rfa de permeabilidad de pared celular y la mutación en el gen uvrB de resistencia a radiación UV). Se realizaron dos tipos de pruebas, la prueba de mutagenicidad para evaluar el efecto genotóxico de la bebida, y la prueba de antimutagenicidad para evaluar la posible inhibición de mutagenicidad por parte de dicha bebida. Ambas pruebas se realizaron siguiendo el protocolo de Maron y Ames (1983). Para detalles del diseńo experimental ver Tabla 1.

En cada caso se evaluó el número de colonias revertantes, se calcularon el índice de mutación (IM) y el porcentaje de inhibición de mutagenicidad (\%de inhibición), con las siguientes fórmulas:

$$
\mathrm{IM}=\frac{\text { \#de colonias revertantes de cada tratamiento }}{\text { \#de colonias revertantes del control negativo }}
$$

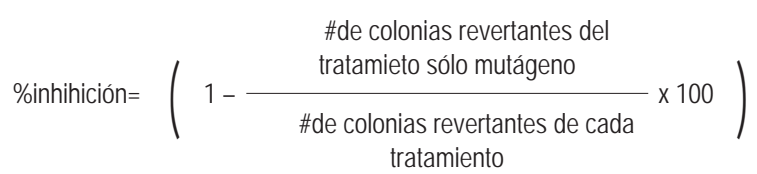

Para el análisis estadístico se utilizó el paquete SPSS v.21 y se aplicó el test de Kolmogorov-Smirnov (W-K) para determinar la normalidad de los datos; luego, una prueba de homogeneidad de varianzas y un análisis de varianza (ANOVA) más una prueba post hoc (Tukey). También se hizo la comparación de los valores obtenidos con datos de otros autores para verificar la viabilidad de las cepas utilizadas y la calidad procedimental (datos no publicados).

Test de micronúcleos.- El test de micronúcleos (MN) fue realizado siguiendo el protocolo modificado por Castillo et al. (2011). Se elaboraron tres ensayos, cultivándose linfocitos, en siete tratamientos: un control negativo, un control positivo (mitomicina C) y la bebida en cinco dosis (10, 100, 200, 400 y $1000 \mu \mathrm{L} /$ tubo de cultivo). El cultivo se mantuvo a $37^{\circ} \mathrm{C} \mathrm{du}-$ rante 72 horas, adicionándose la bebida a evaluar a las 6 horas de cultivo, el mutágeno (control positivo) a las 24 horas y la citocalasina B (inhibidor de la citocinesis para poder observar células binucleadas) a las 40 horas.

La observación y conteo "ciego" se hicieron en un microscopio Zeiss Axioplan con objetivos de 400x y 1000x de aumento. Se calculó el índice de división nuclear (IDN), con un promedio de 500 células por tratamiento, y posteriormente el número de células con MN por cada 1000 células binucleadas y el correspondiente porcentaje, con las siguientes fórmulas:

$$
I D N=\frac{1(1 n)+2(2 n)+3(3 n)+4(4 n)}{1 n+2 n+3 n+4 n}
$$

Donde:

$1 \mathrm{n}=$ \#decélulas con un núcleo,

$2 \mathrm{n}=$ \#de células con dos núcleos,

$3 \mathrm{n}=$ \#de células con tres núcleos,

$4 \mathrm{n}=$ \#de células concon cuatro núcleos. 

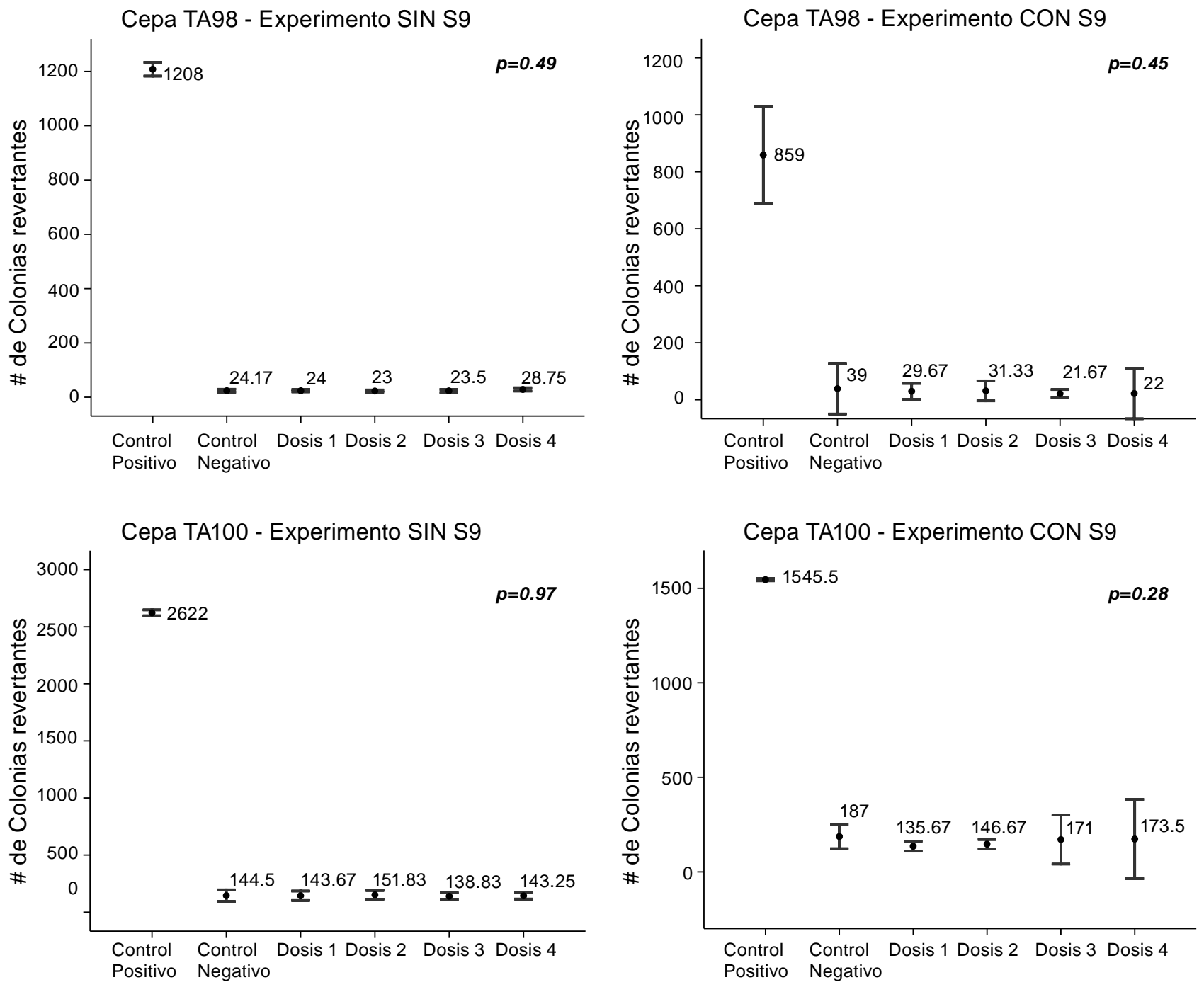

Figura 1. Prueba de mutagenicidad. Promedio de colonias revertantes por tratamiento $(\bullet)$. Desviación estándar en barras verticales. Además, en negrita y en cursiva se muestran los valores de $\boldsymbol{p}$ para ANOVA, entre el control negativo y las diferentes dosis.

$$
\%=\frac{\text { \#de células binucleadasconMNs }}{\text { \#totaldecélulasbinucleadas }}
$$

Para el análisis estadístico, también se utilizó el paquete SPSS v.21, analizando el número de células binucleadas con MN. Se aplicó el test de Kolmogorov-Smirnov para determinar el tipo de distribución de los datos y luego una prueba de comparación de medianas (Kruskal-Wallis) entre el control negativo y las diferentes dosis.

\section{Resultados}

Prueba de Ames.- En la prueba de mutagenicidad, ANOVA indica que no existen diferencias significativas en el número de colonias revertantes entre el control negativo y las cuatro dosis, para ambos experimentos y ambas cepas ( $\mathrm{p}>0.05$ ). (Fig. 1). En la prueba de antimutagenicidad, ANOVA indica que sí existen diferencias significativas entre los tratamientos $(p<0.05)$, y Tukey arroja diferencias entre el tratamiento en el que se usa sólo mutágeno y en los que se usan las dosis más elevadas más mutágeno, para ambas cepas (Fig. 2). Además en las Tablas 2 y 3 se muestran los resultados del índice de mutagenicidad (prueba de mutagenicidad) y el porcentaje de inhibición de la mutagenicidad (prueba de antimutagenicidad), respectivamente.
Test de Micronúcleos.- En la Figura 4 se muestra el índice de división nuclear (IDN), encontrándose en todos los casos entre 1.30 y 1.72. Al analizar el número de células binucleadas con MN por cada mil células binucleadas, los datos no presentan distribución normal ( $\mathrm{p}=0.007)$, así que al contrastar el control negativo con las diferentes dosis, mediante Kruskal-Wallis, se evidencia que no existen diferencias significativas $(\mathrm{p}=0.391)$ entre todos los tratamientos (Fig. 5).

\section{Discusión}

Existe un gran interés por evaluar y validar el uso de compuestos con potencial nutricional y medicinal a fin de brindar a una población en crecimiento, fuentes alternativas de consumo. Ahora se incentiva el uso de alimentos con efectos benéficos, en oposición con aquellos que propician el sobrepeso. Un parámetro poco evaluado en nuestro medio es el potencial genotóxico de algunos compuestos de uso común en las industrias alimentaria, farmacéutica, cosmética, etc., que se distribuyen (en el mercado nacional) sin previos controles. Muchas veces, el riesgo-beneficio así lo justifica, pero en otros casos, es deseable obtener el máximo beneficio con la menor posibilidad de toxicidad, sobre todo, a nivel genético. Esto es importante porque existe una probada conexión entre la mutagenicidad y el proceso de carcinogénesis, 

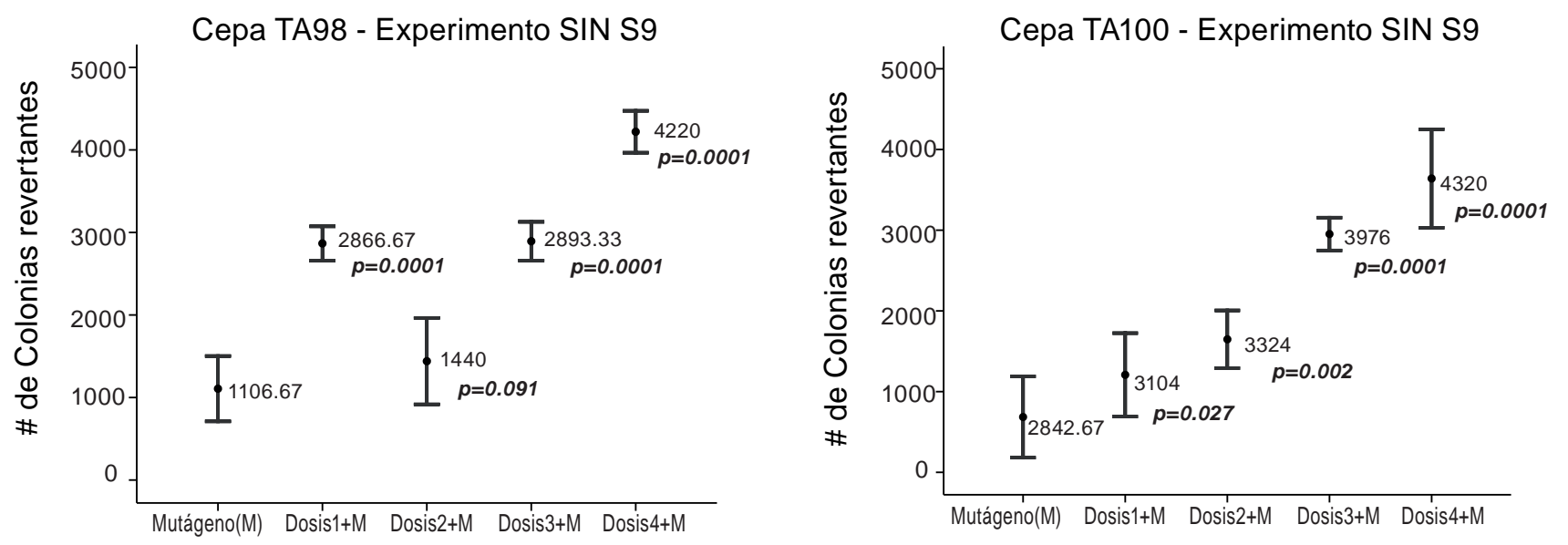

Figura 2. Prueba de antimutagenicidad. Representación gráfica del promedio de colonias revertantes por tratamiento $(\bullet)$. Desviación estándar en barras verticales. Además, en negrita y en cursiva se muestran los valores de $\boldsymbol{p}$, resultantes de las comparaciones múltiples (prueba de Tukey) entre el tratamiento sólo con mutágeno y cada una de las diferentes dosis.
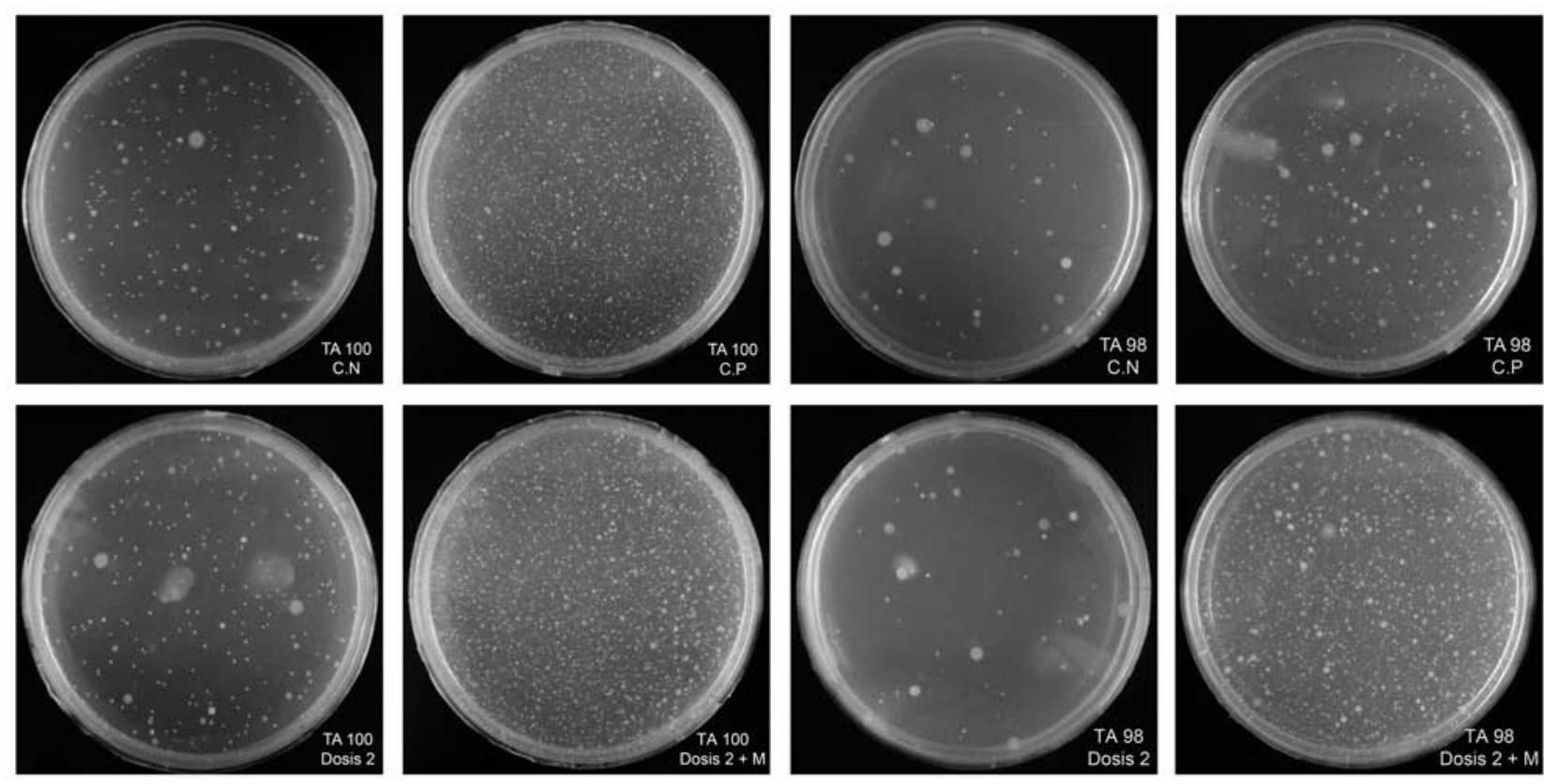

Figura 3. Resultados de la prueba de Ames. Se muestran las fotografías de las placas con crecimiento (colonias revertantes) de la Salmonella typhimurium, cepas TA98 y TA100, CN (control negativo), CP (control positivo) y M (mutágeno).

Tabla2. Resultados de la prueba de mutagenicidad

\begin{tabular}{lccccc}
\hline \multirow{2}{*}{ Cepa } & \multicolumn{3}{c}{ Índice de mutagenicidad } \\
\cline { 2 - 3 } Experimento & \multicolumn{2}{c}{ Ta 98 } & & \multicolumn{2}{c}{ Ta 100 } \\
\cline { 2 - 3 } \cline { 5 - 6 } Tratamiento & Sin S9 & Con S9 & & Sin S9 & Con S9 \\
CP & 49.99 & 22.03 & & 18.15 & 8.26 \\
CN & 1.00 & 1.00 & & 1.00 & 1.00 \\
Dosis1 & 0.99 & 0.76 & & 0.99 & 0.73 \\
Dosis2 & 0.95 & 0.80 & & 1.05 & 0.78 \\
Dosis3 & 0.97 & 0.56 & & 0.96 & 0.91 \\
Dosis4 & 1.19 & 0.56 & & 0.99 & 0.93 \\
\hline
\end{tabular}

Tabla3. Resultados de la prueba de antimutagenicidad.

\begin{tabular}{lcc}
\hline & \multicolumn{2}{c}{$\begin{array}{c}\text { Porcentaje de inhibición de la } \\
\text { mutagenicidad }\end{array}$} \\
\cline { 2 - 3 } Experimento & \multicolumn{2}{c}{ Sin S9 } \\
\cline { 2 - 3 } Cepa & TA 98 & TA 100 \\
\hline Tratamiento & 0 & 0 \\
Mutágeno (M) & -137.31 & -18.27 \\
Dosis1 + M & -19.21 & -26.77 \\
Dosis2 + M & -139.51 & -51.64 \\
Dosis3 + M & -249.34 & -64.76 \\
Dosis4 + M & & \\
\hline
\end{tabular}




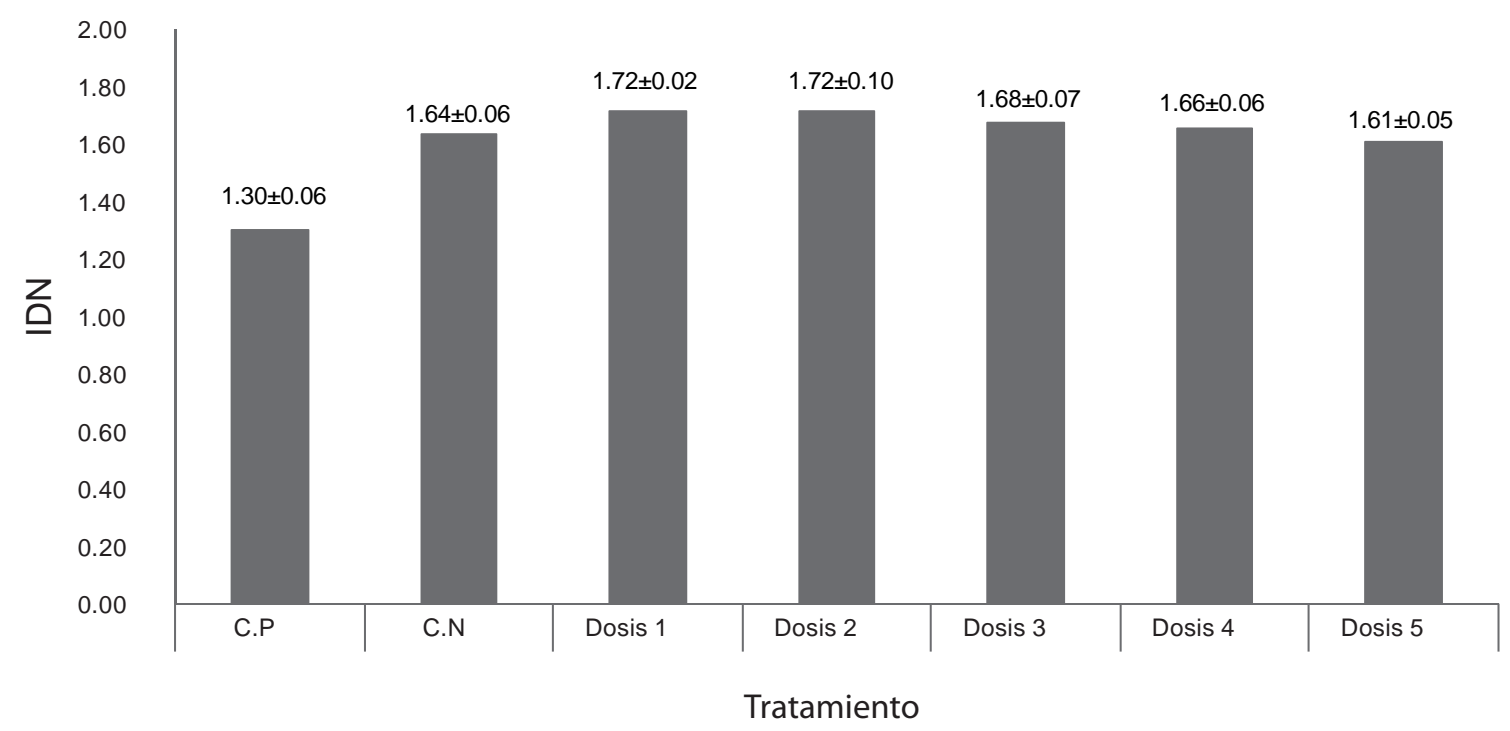

Figura 4. Prueba de micronúcleos. Representación del índice de división nuclear (IDN). Donde, CP: control positivo, CN: control negativo, Dosis 1, 2, 3, 4 y 5 corresponden a 10, 100, 200, 500, $1000 \mu \mathrm{L}$ de bebida respectivamente.

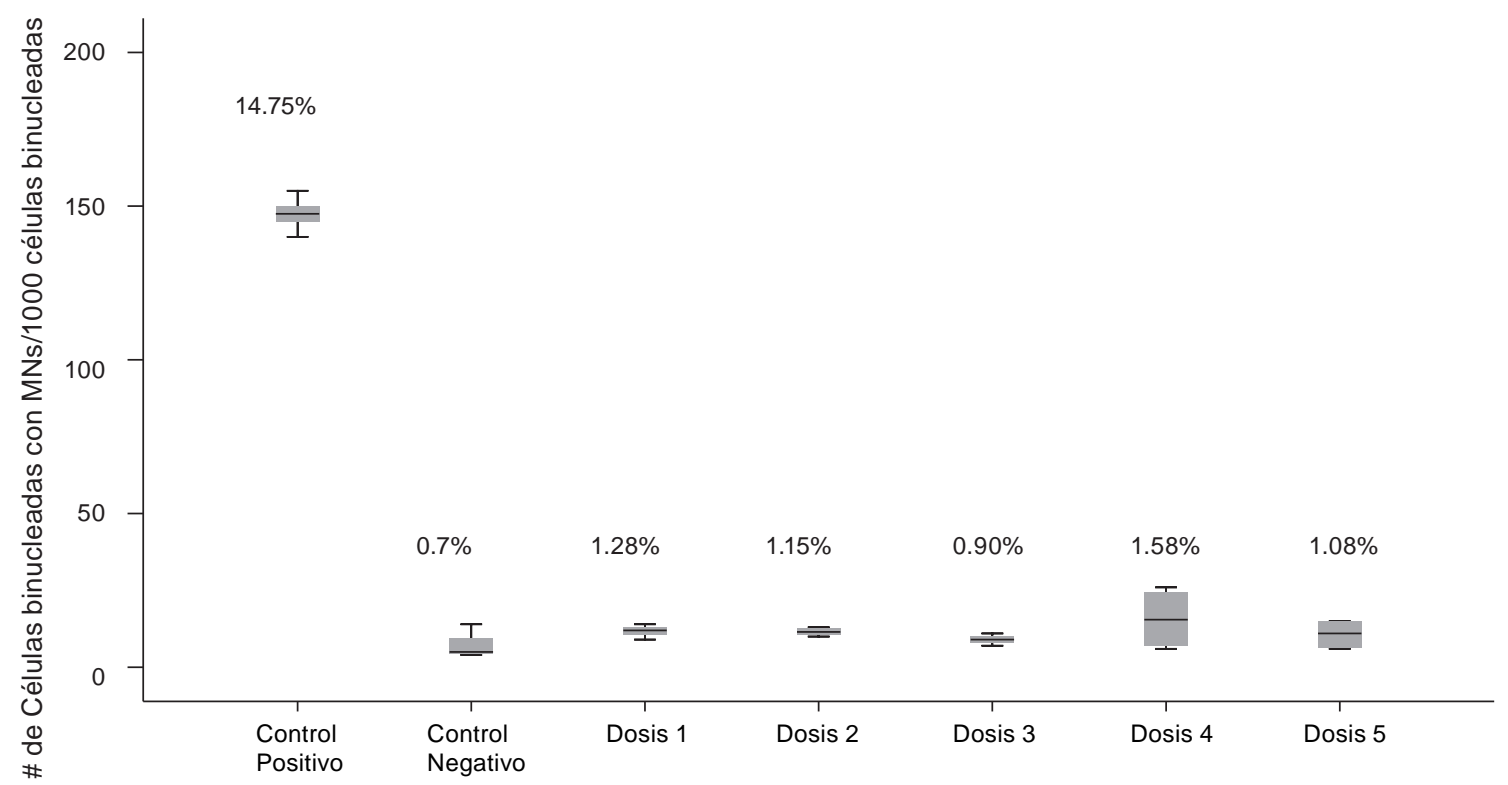

Figura 5. Prueba de micronúcleos. Representación gráfica de la mediana. Se muestran los valores del porcentaje de células binucleadas con micronúcleos.

y que en exposiciones crónicas puede tener un efecto aditivo (Gupta et al. 2010, Déciga-Campos et al. 2007).

Para evaluar la genotoxicidad, se requieren dos pruebas in vitro con resultados positivo o negativo, para determinar el potencial efecto genotóxico de un componente, y si los resultados difieren o no son concluyentes, se puede realizar una prueba adicional. (U.S Department of Health and Human Services Food and Drug Administration, CDER y CBER 2012). Además, cada prueba tiene sus fortalezas y debilidades por lo que un solo ensayo no determina con seguridad, el potencial mutagénico (Srividye et al. 2013).

En el presente estudio, la prueba de mutagenicidad (prueba de Ames), indica que para ambas cepas (TA98 y TA100) y tanto en experimentos con y sin actividad metabólica, no se presentan diferencias significativas entre el control negativo y las distintas dosis en relación al número de colonias revertantes (Figs. 1 y 3). Además, el índice de mutación es menor a dos en todos los tratamientos con dosis (Tabla 2), por lo que según el Cornell Institute for Medical Research (1986) se encuentra en el rango de lo normal, lo que implicaría que la bebida es inocua bajo esas condiciones. Sin embargo, en la prueba de antimutagenicidad, donde se evalúa el posible efecto protector (porcentaje de inhibición de la mutagenicidad) de la bebida ante mutágenos ya establecidos, los resultados de las comparaciones múltiples indican diferencias significativas entre el tratamiento sólo mutágeno y los demás tratamientos que contienen las dosis más elevadas más mutágeno, lo que indicaría que al añadir la bebida, aumenta el efecto mutagénico del NPD y la azida de sodio (para TA98 y TA100 respectivamente). (Figs. 2 y 3 , y Tabla 3). 
Cabe mencionar que en la Figura 2, se observa entre los promedios del número de revertantes, una tendencia en aumento más evidente en la cepa TA100 en comparación a la cepa TA98. Esto podría deberse a que la bebida presenta mayor eficiencia en la reversión de la mutación del tipo de sustitución de pares de bases (TA100) que la de cambio del marco de lectura (TA98). En cualquier caso, se observa la tendencia de incremento de revertantes a medida que la dosis de bebida aumenta, lo que se refleja en que el porcentaje de inhibición de la mutagenicidad para todos los casos de dosis más mutágeno es menor a cero, según el Cornell Institute for Medical Research (1986).

Estos resultados, indican la necesidad de hacer estudios similares de los componentes de la bebida por separado, e intentar explicar por qué, cuando se mezclan, resulta el efecto aquí descrito en presencia de mutágenos.

Por otro lado en el análisis de micronúcleos, se usaron los criterios establecidos por HUMN, grupo de trabajo que publicó la estandarización de la técnica a partir de una base de datos de varios laboratorios del mundo (teniendo en cuenta los criterios de análisis, variantes de protocolos, etc.), analizándose en conjunto unos 7000 individuos (Bonassi et al. 2001, Fenech et al. 2003). En el presente estudio, los valores del IDN (Fig. 4), en todas las dosis de bebida están dentro de los parámetros establecidos por HUMN (1.38 - 1.95) (Bonassi et al. 2001). A partir de esto podemos afirmar que la bebida no presenta un efecto inhibidor del crecimiento celular en relación con el control negativo.

Sobre la evaluación del porcentaje de células binucleadas con micronúcleos, se muestra que el control positivo (14.75\%) es el único tratamiento con valor por encima del rango normal (3-12\%), reportado por HUMN (Bonassi et al. 2001), lo que demostraría que las diferentes dosis $(0.7-1.58 \%)$ no presentan efecto genotóxico. Según Kruskal-Wallis, los valores de las medianas del número de células con MN por cada 1000 células binucleadas, no muestran diferencias significativas, demostrándose que la bebida no es genotóxica según este ensayo.

Entonces, tenemos los resultados de dos pruebas in vitro (prueba de mutagenicidad de Ames y la prueba de MN) que nos indican que la bebida es aparentemente inocua. Sin embargo, es importante considerar los resultados de la prueba de antimutagenicidad de Ames, que parece revelar un efecto pro-mutagénico, debido a un mecanismo aditivo o sinérgico de uno o de los tres pseudocereales con el mutágeno. Debemos resaltar que, es conocido que algunos compuestos aislados de plantas, como la quercitina, furoquinolina, alcaloides e isotiocianatos, presentan un riesgo mutagénico. (Déciga-Campos et al. 2007, Verschaeve \& Van Stander 2008, Srividye et al. 2013). Es difícil determinar qué compuesto es mutagénico en una mezcla compleja y en algunos casos se ha determinado que pueden potenciar el efecto de otros xenobióticos, de ahí la importancia de la evaluacion de plantas que tienen uso medicinal (Srividye et al. 2013).

En conclusión, en base a los resultados de las pruebas de mutagenicidad, con dos pruebas in vitro negativas, se podría validar su consumo, sin embargo se recomienda realizar pruebas adicionales in vitro, en las que se evalúen: la bebida, cada uno de sus compuestos por separado y combinaciones de a dos; y también pruebas in vivo bajo distintos diseños experimentales, sobre todo a largo plazo, para confirmar o descartar estos resultados iniciales. Esto aunque las dosis que se emplearán sean bajas y el periodo de exposición a la bebida sea corto, porque es importante descartar cualquier posible efecto secundario debido a su consumo.

\section{Agradecimientos}

Al doctor Ricardo M. Fujita, por la revisión del manuscrito. A la doctora Berta Loja por la verificación de la taxonomía de pseudocereales empleados en la elaboración de la bebida. A la USMP y al CONCYTEC (Proy. 368-2012-OAJ) por su apoyo logístico y financiero.

\section{Literatura citada}

Altmann S., H. Davis, J. Zhu, et al. 2004. Niemann-Pick C1 Like 1 Protein is critical for intestinal cholesterol absorption. Science. 303: 1201-4. http://dx.doi.org/10.1126/science. 1093131

Arencibia D.F. \& L.A. Rosario. 2009. Actualización sobre el ensayo cometa y de micronúcleos in vitro. Retel,revista de toxicología en línea no20 [septiembre 2009] pp:18. < http://www. sertox.com.ar/img/item_full/20003.pdf> [Acceso: ¿]

Bonassi S., M. Fenech, C. Lando, et al. 2001. HUman MicroNucleus Project: International Database Comparison for Results With the Cytokinesis-Block Micronucleus Assay in Human Lymphocytes: I. Effect of Laboratory Protocol, Scoring Criteria, and Host Factors on the Frequency of Micronuclei. Environmental and Molecular Mutagenesis 37:31-45. http://dx.doi.org/10.1002/1098-2280(2001)37:1<31::AIDEM1004>3.0.CO;2-P

Carrasco R., J. Hellstrom, J. Pihlava, et al. 2010. Flavonoids and other phenolic compounds in Andean indigenous grains: Quinoa (Chenopodium quinoa), kañiwa (Chenopodium pallidicaule) and kiwicha (Amaranthus caudatus). Food Chemistry. 120: 128-133. http://dx.doi.org/10.1016/j. foodchem.2009.09.087

Castillo E., M.L. Guevara-Fujita \& R. Fujita. 2011. Optimización del test de micronúcleos en linfocitos cultivados usando una metodología de gradiente y frotis. Revista Peruana Biología 18 (2): 261-263. http://dx.doi.org/10.15381/rpb.v18i2.241

Claxton LD, J. Allen, A. Auletta, et al. 1987. Guide for the Salmonella typhimurium/mammalian microsome tests for bacterial mutagenicity. Mutation Research 189:83-91. http://dx.doi. org/10.1016/0165-1218(87)90014-0

Cornell Institute for Medical Research. 1986. Ames Salmonella mutagenicity assay protocol. Departament of microbiology. Camden. New Jersey 08103

Déciga-Campos M., I. Rivero-Cruz, M. Arriaga-Alba, et al. 2007. Acute toxicity and mutagenic activity of Mexican plants used in traditional medicine. Journal of Ethnopharmacology 110, 334-342. http://dx.doi.org/10.1016/j.jep.2006.10.001

European Medicines Agency. 1996. Note for guidance on genotoxicity: specific aspects of regulatory genotoxicity tests for pharmaceuticals (CPMP/ICH/141/95) (Approval by the Committee for proprietary medical products (CPMP) y the International Conference on Harmonisation (ICH)). http:// www.ema.europa.eu/docs/en_GB/document_library/Scientific_guideline/2009/09/WC500003146.pdf [Acceso: ¿]

Fenech M., W.P. Chang, M. Kirsch-Volders, et al. 2003. HUMN proyect: detailed description of the scoring criteria for the cytokinesis-block micronucleus assay using isolated human lymphocyte cultures. Mutation Research 534, 65-75. http:// dx.doi.org/ 10.1016/S1383-5718(02)00249-8

Gálvez, L., E. Apostolidis, M. Genovese, et al. 2009. Evaluation of indigenous grains from the Peruvian Andean Region for antidiabetes and antihypertension using in vitro methods. Journal of Medical Food. 12(4): 704-13. http://dx.doi. org/10.1089/jmf.2008.0122.

Gupta S.C., J.H. Kim, S. Prasad, et al. 2010. Regulation of survival, proliferation, invasion, angiogenesis, and metastasis of tumor cells through modulation of inflammatory pathways by nutraceuticals. Cancer and Metastasis Reviews 29:405-434. http://dx.doi.org/10.1007/s10555-010-9235-2.

Huff M. 2003. Dietary cholesterol, cholesterol absorption, postprandial lipemia and atherosclerosis. Canadian Journal of Clinical Pharmacology. 10: A: 26A-32A. 
Maron D.M. \& B.N. Ames. 1983. Revised methods for the Salmonella mutagenicity test. Mutation Research, 113, 173-215. http:// dx.doi.org/10.1016/0165-1161(83)90010-9

Mortelmans K. \& E. Zeiger. 2000. The Ames Salmonella / microsome mutagenicity assay. Mutation research 455, 29-60. http:// dx.doi.org/10.1016/S0027-5107(00)00064-6

Rastrelli L., P. Saturnino, O. Schettino, et al. 1995. Studies on the constituents of Chenopodium pallicaule (Cañihua) seeds; isolation and characterization of two new flavonol glycosides. Journal of Agricultural and Food Chemistry. 43(8): 2020-4. http://dx.doi.org/10.1021/jf00056a012

Rossi R., T. Grimaldi, G. Origliani, et al. 2002. Menopause and cardiovascular risk. Pathophysiology of Haemostasis and Thrombosis 32: 325-328. http://dx.doi.org/10.1159/000073591

Srividya A.R., S.P. Dhanabal, M.N. SathishKumar,et al. 2013. Genoprotective Activity of hydro alcoholic extract of Curcuma aromatic and Curcuma zedoaria rhizomes and their comparison with Curcumin. The Journal of Ethnobiology and Traditional Medicine. Photon. 118: 306-321.

Tsuda, T.; Horio, F.; Uchida, K.; et al. 2003. Dietary cyaniding 3-Ob-d-glucoside-rich purple corn color prevents obesity and ameliorates hyperglycemia in mice. The Journal of Nutrition 133(7):2125-2130.

Turley S. \& J. Dietschy. 2003. Sterol absorption by the small intestine. Current Opinion in Lipidology. 14(3): 233-40.
U.S Department of Health and Human Services Food and Drug Administration, Center for Drug Evaluation and Research (CDER) \& Center for Biologics Evaluation and Research (CBER). 2012. Guidance for Industry. S2 (R1) Genotoxicity Testing and Data Interpretation for Pharmaceuticals Intended for Human Use (Approval by the International Conference on Harmonisation (ICH)). Pp31. http://www. fda.gov/downloads/Drugs/Guidances/ucm074931.pdf

U.S. Environmental Protection Agency. 2010. Toxic Substances Control Act - New Chemical Program. http://www.epa. gov/oppt/newchems/

Verschaeve L. \& J. Van Staden, 2008. Mutagenic and antimutagenic properties of extracts from South African traditional medicinal plants. Journal of Ethnopharmacology 119, 575-587. http://dx.doi.org/10.1016/j.jep.2008.06.007

Zalacain M., L. Sierrasesúmaga \& A. Patińo. 2005. El ensayo de micronúcleos como medida de inestabilidad genética inducida por agentes genotóxicos. Anales del Sistema Sanitario de Navarra 28 (2): 227-236. http://dx.doi.org/10.4321/ S1137-66272005000300007 